\title{
Evidências de Validade da Escala de Flexibilidade Psicológica no Trabalho em Amostras Brasileiras
}

\author{
Vladimir Pinto Novaes \\ Maria Cristina Ferreira \\ Felipe Valentini \\ Universidade Salgado de Oliveira \\ Rio de Janeiro, RJ, Brasil
}

\begin{abstract}
RESUMO
A flexibilidade psicológica no trabalho diz respeito à capacidade de o indivíduo adotar ações direcionadas à consecução de suas metas laborais, ainda que na presença de experiências internas de difícil aceitação. O estudo objetivou adaptar e reunir evidências de validade da Escala de Flexibilidade Psicológica no Trabalho (WAAQ) no contexto brasileiro. A amostra foi composta por 583 trabalhadores, de ambos os sexos, com idades variando de 18 a 62 anos. As análises fatoriais confirmatórias evidenciaram que a versão brasileira da escala reproduziu integralmente a estrutura original do instrumento. A escala apresentou, ainda, correlações positivas com o engajamento no trabalho, a saúde mental, a abertura à experiência e a satisfação com a vida, além de uma correlação negativa com o neuroticismo. Concluiu-se que a WAAQ apresentou características psicométricas adequadas, o que recomenda seu uso em situações futuras de pesquisa da flexibilidade no contexto do trabalho.
\end{abstract}

Palavras-chave: Psicometria; Saúde Mental; Trabalho; Traços de Personalidade.

\begin{abstract}
Validity Evidences of the Work Acceptance and Action Questionnaire in Brazilian Samples

The psychological flexibility at work is related to the ability of the individuals to take work goal directed actions, even in the presence of difficult internal experiences. The study aimed to adapt and gather evidences of validity of the Scale of Psychological Flexibility at Work (WAAQ) in the Brazilian context. The sample consisted of 583 employees, of both sexes, aged 18-62 years. Confirmatory factor analyzes showed that the Brazilian version of the scale reproduced fully the original structure of the instrument. The scale has also presented positive correlations with job engagement, mental health, openness to experience and life satisfaction, as well as a negative correlation with neuroticism. It was concluded that the WAAQ showed adequate psychometric properties and can therefore be used in the future research of flexibility at the workplace.
\end{abstract}

Keywords: Psychometrics; Mental Health; Work; Personality Traits.

\section{RESUMEN}

Evidencias de Validad de la Escala de Flexibilidad Psicologíca en el Trabajo en Muestras Brasileñas

La flexibilidad psicológica en el trabajo es la capacidad del individuo adoptar acciones direccionadas a la consecución de sus objetivos laborales, todavía en presencia de experiencias internas de difícil aceptación. El estudio buscó adaptar y reunir evidencias de validez de la Escala de Flexibilidad Psicológica en el Trabajo (WAAQ) en el contexto brasileño. La muestra está formada por 583 empleados, de ambos sexos, de edades 18 a 62 años. Las análisis factoriales confirmatorios demostraron que la versión brasileña de la escala reproducís totalmente la estructura original del instrumento. La escala presentó correlaciones positivas con el compromiso en el trabajo, la salud mental, la apertura a la mudanza y la satisfacción con la vida, y correlación negativa con el neuroticismo. La conclusión es que la WAAQ mostró propiedades psicométricas adecuadas y se puede utilizar en las investigaciones futuras de la flexibilidad en el trabajo.

Palabras clave: Psicomteria; Salud Mental; Trabajo; Rasgos de Personalidad. 
Ao início do século XX, ganha força o movimento da Psicologia Positiva, que critica a abordagem tradicional de analisar a patologia do funcionamento humano e defende uma abordagem relacionada principalmente à qualidade de vida e ao bem-estar (Seligman \& Csikszentmihalyi, 2000). No contexto individual, a Psicologia Positiva dedicase ao estudo dos traços individuais positivos, como por exemplo, a capacidade de amar, a coragem, a perseverança e o perdão. Alinhado com tal perspectiva, o presente trabalho tem como foco um traço individual positivo, qual seja a flexibilidade psicológica.

A flexibilidade psicológica pode ser conceituada como a capacidade de contato pleno e consciente com o momento presente, o que permite ao indivíduo persistir ou modificar o curso de suas ações para atingir seus valores e metas, mesmo quando na presença de pensamentos ou sentimentos desafiadores ou indesejáveis (Hayes, Luoma, Bond, Masuda, \& Lillis, 2006). Ela capacita, portanto, as pessoas a se comportarem de acordo com seus valores e metas, isto é, com foco em seus objetivos e não em suas experiências internas ou contingências situacionais momentâneas (Bond et al., 2011). No contexto do trabalho, a flexibilidade psicológica diz respeito à capacidade de emissão de comportamentos condizentes com a consecução das metas laborais, ainda que na presença de experiências internas de difícil aceitação, como, por exemplo, a ansiedade de cometer erros (Bond, Lloyd, \& Guenole, 2013).

Os estudos nessa área vêm utilizando o Questionário de Ação e Aceitação (AAQ-I; Hayes et al., 2004) e sua versão revisada, o AAQ-II (Bond et al., 2011), para avaliação dos níveis gerais de flexibilidade psicológica. Mais recentemente, porém, Bond et al. (2013) desenvolveram o Questionário de Ação e Aceitação no Trabalho (WAAQ), adaptado posteriormente para amostras espanholas (Ruiz \& Odriozola-González, 2014), fundamentando-se na premissa de que o referido instrumento poderia se mostrar mais útil ao estudo das associações do construto com variáveis especificas ao contexto laboral.

No entanto, os estudos nacionais e estrangeiros com foco na flexibilidade psicológica no contexto do trabalho ainda são incipientes, na medida em que apenas mais recentemente o referido construto foi introduzido na literatura da área organizacional. Contudo, as investigações sobre tal fenômeno mostramse relevantes, na medida em que a flexibilidade psicológica em geral tem se mostrado determinante da saúde mental (Bond \& Bunce, 2000; Kashdan \& Rottenberg, 2010).
Nesse sentido, a investigação da flexibilidade no contexto do trabalho poderá contribuir para a criação de ambientes mais saudáveis e propícios à realização pessoal, o que, por sua vez, poderá impactar diversos comportamentos organizacionais, como por exemplo, a rotatividade, o absenteísmo e o desempenho. Apoiandose nessas considerações, e no fato de a consulta às bases de dados nacionais (PePSIC; Scielo) terem revelado a inexistência de instrumentos desenvolvidos e validados com tal propósito, o presente estudo teve como objetivo adaptar e reunir evidencias de validade da Escala de Avaliação da Flexibilidade Psicológica no Ambiente do Trabalho (WAAQ; Bond et al., 2013), em amostras brasileiras.

\section{A FLEXIBILIDADE PSICOLÓGICA}

Segundo Kashdan e Rotterburg (2010), a flexibilidade psicológica pode ser definida como uma medida de como as pessoas se adaptam às demandas situacionais flutuantes, reconfigurando seus recursos mentais e deslocando perspectivas, desejos conflitantes e necessidades, com o intuito de se comportarem de forma condizente com seus valores e metas. As pessoas com maior flexibilidade psicológica possuem, assim, maior capacidade de se concentrar na situação atual e, com base nas características de tal situação, comportar-se de modo adequado à consecução de seus objetivos e valores, ainda que a referida situação seja psicologicamente desafiadora ou indesejada (Hayes, Levin, Plumb-Vilardaga, Villatte, \& Pistorello, 2013).

Em outras palavras, quanto mais desenvolvida for a flexibilidade psicológica, mais apto estará o indivíduo a reconfigurar seu estado mental e otimizar seus recursos psicológicos, assim como a cumprir as exigências e os desafios do dia-a-dia e se adaptar às situações, de modo a emitir ações que lhe permitam o alcance de seus objetivos (Kashdan \& Rottenberg, 2010). As pessoas psicologicamente flexíveis comportam-se portanto muito mais em função de seus valores do que de suas experiências internas ou contingências situacionais momentâneas (Bond et al., 2011). Por essa razão, a flexibilidade psicológica é considerada uma das principais determinantes da saúde mental (Flaxman \& Bond,2010).

O construto flexibilidade psicológica surge no bojo da Teoria da Terapia da Aceitação e Compromisso (ACT; Hayes, Strosahl, \& Wilson, 1999). De acordo com essa teoria, seis processos-chave são importantes para o desenvolvimento da flexibilidade psicológica: a aceitação, que envolve a tomada de consciência dos eventos sobre si próprio na forma em que eles são, isto é, sem a tentativa de modificá-los; a desfusão 
cognitiva, que consiste na alteração da forma com que o indivíduo interage com seus pensamentos, visando modificar as funções indesejáveis desses pensamentos; estar presente, que diz respeito à possibilidade de contato com os eventos psicológicos e ambientais sem julgamentos prévios; o eu em contexto, que se refere à tomada de contato com as próprias experiências sem uma ligação muito forte com elas; valores, que se associam à busca de direções e escolhas na vida sem evitação; e a ação comprometida, que diz respeito ao desenvolvimento de padrões de ações coerentes com os valores adotados. Tomados em conjunto, tais processos objetivam, em última análise, o contato do indivíduo com o momento presente como uma pessoa consciente, que persiste ou muda seu comportamento de acordo com os valores por ela adotados (Hayes et al., 2013).

Para avaliar a flexibilidade psicológica foi desenvolvido o Questionário de Ação e Aceitação (AAQ-I; Hayes et al., 2004), tomando-se por base a ACT. $\mathrm{O}$ instrumento contém itens relacionados às avaliações negativas de sentimentos (por exemplo, "ansiedade é ruim") e à evitação de pensamentos e sentimentos (por exemplo, "eu tento suprimir pensamentos e sentimentos que eu não gosto, simplesmente não pensando neles").

$\mathrm{O}$ referido instrumento apresentou, no entanto, problemas relacionados a seus índices de consistência interna e de complexidade dos itens, o que levou ao desenvolvimento de uma segunda versão do questionário, chamada de AAQ-II (Bond et al., 2011), a qual tem por base o AAQ-I. Essa segunda versão contém itens que refletem a falta de vontade de experimentar emoções e pensamentos indesejáveis (por exemplo, "Eu tenho medo dos meus sentimentos"), a capacidade de estar no momento presente (por exemplo, "Eu estou no controle da minha vida") e o compromisso com ações e valores voltados à experimentação de eventos psicológicos, sem que pensamentos indesejáveis o atrapalhem (por exemplo, "Meus pensamentos e sentimentos não ficam no caminho de como eu quero viver minha vida"). As propriedades psicométricas do AAQ-II mostraram-se mais apropriadas que as do AAQ-I (Bond et al., 2011).

\section{A FLEXIBILIDADE PSICOLÓGICA NO CONTEXTO DO TRABALHO}

A flexibilidade psicológica no trabalho diz respeito à capacidade de o indivíduo emitir comportamentos condizentes com a consecução de suas metas laborais, ainda que na presença de experiências internas de difícil aceitação (como, por exemplo, o medo de tomar iniciativa), sem defesas ou evasões desnecessárias (Bond et al., 2013). Nesse sentido, indivíduos com maior flexibilidade psicológica mostram-se mais capazes de responder com eficácia às contingências do contexto laboral que costumam impedir a realização de metas e, consequentemente, apresentar maior motivação, satisfação e engajamento no trabalho, além de maiores índices de desempenho, bem como menores taxas de absenteísmo (Bond et al., 2013; Ruiz \& Odriozola-González, 2014).

Com o objetivo de avaliar a flexibilidade psicológica relacionada ao ambiente de trabalho, foi desenvolvido o Questionário de Ação e Aceitação no Trabalho (WAAQ; Bond et al., 2013), também ancorado na Teoria ACT (Hayes et al., 1999). O instrumento inicial era composto de 32 itens, que representavam as maneiras pelas quais a flexibilidade psicológica podia se manifestar no local de trabalho, especialmente na presença de experiências difíceis como, por exemplo, admitir os próprios erros e ainda assim ser bem sucedido. Os resultados das análises fatoriais exploratórias e confirmatórias levaram a uma versão final da escala composta por sete itens, que apresentou boa consistência interna $(\alpha=0,84)$ (Bond et al.,2013).

Diferentes estudos têm demonstrado que a flexibilidade psicológica caracteriza-se como importante promotora do bem-estar e da saúde mental no ambiente de trabalho (Bond et al., 2013), em função de as pessoas mais flexíveis experimentarem níveis mais elevados de motivação e de foco nas tarefas (Bond, Flaxman, van Veldhoven, \& Biron, 2010). Bond e Bunce (2000), por sua vez, em intervenção focada no aumento da flexibilidade psicológica como forma de reduzir os problemas de saúde mental no local de trabalho, constataram que a intervenção melhorou significativamente a saúde mental em geral, assim como os sintomas depressivos.

Fundamentando-se em tais considerações, a presente pesquisa objetivou buscar evidências sobre a validade de construto da Escala de Avaliação da Flexibilidade Psicológica no Ambiente do Trabalho, mediante a adoção de procedimentos de análise fatorial confirmatória e da correlação da escala com medidas de construtos correlatos à flexibilidade psicológica. Nesse sentido, foram utilizadas medidas de engajamento no trabalho, saúde mental, abertura, neuroticismo e satisfação com a vida.

Considerando que o engajamento no trabalho refere-se a um estado afetivo-motivacional de bemestar relacionado ao trabalho, manifesto em sentimentos de vigor, de dedicação e absorção (Bakker, Demerouti, \& Sanz-Vergel, 2014), seria de se esperar que a flexibilidade psicológica se mostrasse positivamente associada tanto ao engajamento no 
trabalho, quanto a maiores índices de saúde mental. A abertura consiste em uma das facetas do modelo dos cinco grandes fatores da personalidade (CGF; John \& Srivastava, 1999), sendo que pessoas com maiores níveis dessa característica tendem a ser mais proativas, curiosas, criativas e com interesses amplos, além de demonstrarem maior apreciação da experiência por si só (Friedman \& Schustack, 2004). Logo, seria de se esperar que a flexibilidade psicológica se apresentasse positivamente associada também à abertura. A escala de satisfação com a vida tem como objetivo uma avaliação global da qualidade de vida e da boa saúde mental de uma pessoa, de acordo com os critérios por ela escolhidos (Diener, Emmons, Larsen, \& Griffin, 1985), o que levaria à expectativa de que as pessoas com maior nível de flexibilidade psicológica apresentassem maiores níveis de satisfação com a vida. O neuroticismo, por sua vez, também é um componente do modelo dos cinco grandes fatores da personalidade (CGF) e associa-se a características tais como a tensão, o mau humor e à baixa autoestima (Friedman \& Schustack, 2004). Seria assim de se esperar que a flexibilidade psicológica apresentasse uma correlação negativa com o neuroticismo.

\section{MÉTODO}

\section{Participantes}

A amostra foi selecionada por conveniência e o único critério de inclusão referiu-se ao fato de a pessoa trabalhar há pelo menos um ano. Ela foi composta por 583 trabalhadores de organizações públicas e privadas, provenientes das cidades de Niterói e do Rio de Janeiro, que concordaram voluntariamente em participar da pesquisa. A maioria dos participantes era de sexo feminino $(64,5 \%)$ e suas idades variaram de 18 a 62 anos $(M=32,94 ; D P=10,08)$. Quanto ao estado civil, $54,4 \%$ eram solteiros e $38,1 \%$, casados; os demais participantes $(7,5 \%)$ eram separados ou viúvos. No que diz respeito à escolaridade, 52,7\% possuíam nível superior incompleto e $43,2 \%$, nível superior completo; os demais participantes $(4,1 \%)$ possuíam ensino médio completo e/ou técnico. O tempo de trabalho dos participantes na sua empresa atual variou de 1 a 35 anos $(M=5,84 ; D P=6,80)$, enquanto o tempo de trabalho total variou de 1 a 40 anos $(M=11,97 ; D P=9,63)$. No que tange à função desempenhada, 28,3\% pertenciam ao nível administrativo, $23,8 \%$ atuavam na função de supervisor e $16 \%$, na função de gerente de nível médio; os demais participantes $(31,9 \%)$ eram diretores $(5,1 \%)$ ou desempenhavam funções diversas. No que se refere à renda, a maior parte $(46,9 \%)$ recebia entre um e três salários mínimos, e apenas $9,9 \%$ afirmou receber mais de dez salários mínimos; os demais participantes $(43,2 \%)$ recebiam entre três e dez salários.

\section{Instrumentos}

Para a avaliação da flexibilidade psicológica no trabalho foi utilizada a Escala de Avaliação da Flexibilidade Psicológica no Ambiente do Trabalho (WAAQ; Bond et al., 2013), que se compõe de sete itens (por exemplo: Posso admitir meus erros no trabalho e ainda assim ser bem sucedido; Posso trabalhar de forma eficaz, mesmo quando duvido de mim mesmo), a serem respondidos em escalas tipo Likert de sete pontos, variando em função do grau em que apresentam determinados sentimentos em relação a seu dia a dia no trabalho. A tradução dos itens da escala do inglês para o português foi conduzida com o uso da técnica de tradução-retradução. Nesse sentido, inicialmente os itens foram traduzidos para o português e retraduzidos para o inglês por tradutores independentes, após o que as duas versões foram comparadas e os acertos na escala em português realizados.

O engajamento no trabalho foi avaliado pela versão reduzida da Escala de Engajamento no Trabalho (UWES), desenvolvida por Schaufeli e Bakker (2003) e adaptada para amostras brasileiras por Ferreira et al. (2014). Ela se compõe de nove itens (por exemplo: Em meu trabalho, sinto-me repleto (cheio) de energia; Estou entusiasmado com meu trabalho), em formato de resposta tipo Likert de sete pontos. No presente estudo, o coeficiente de consistência interna da escala, calculado pelo coeficiente Alfa de Cronbach, foi igual a 0,91 .

Para a avaliação da saúde mental foi utilizada a versão abreviada do Questionário de Saúde Geral de Goldberg (1972), adaptado para o Brasil por Pasquali, Gouveia, Andriola, Miranda e Ramos (1994), o qual se compõe de 12 itens (por exemplo: Tem se sentido pouco feliz e deprimido? Tem perdido a confiança em si mesmo?), respondidos em escalas do tipo Likert de quatro pontos. A consistência interna da escala, no presente estudo, foi igual a 0,81 .

Os fatores de personalidade abertura à experiência e neuroticismo foram mensurados por duas subescalas do Inventário dos Cinco Grandes Fatores da Personalidade (IGFP-5), elaborado originalmente por John, Donahue e Kentle (1991), e adaptado ao contexto brasileiro por Andrade (2008). A escala de abertura é composta por nove itens, a serem respondidos em escalas do tipo Likert de cinco pontos. Exemplo de item: Eu me vejo como alguém que é original, tem sempre novas ideias. A escala de neuroticismo compõe-se de seis itens, a serem também respondidos em escalas tipo Likert de cinco pontos. Exemplo de item: Eu me vejo como 
alguém que é emocionalmente estável, não se altera facilmente. Os coeficientes de precisão dessas escalas, na presente pesquisa, foram respectivamente iguais a 0,71 e 0,76 .

Para avaliação da satisfação com a vida, adotouse a escala originalmente proposta por Diener et al. (1985), e adaptada ao contexto brasileiro por Gouveia, Barbosa, Andrade e Carneiro (2005). A escala compõe-se de cinco itens (por exemplo: Estou satisfeito com minha vida; As condições da minha vida são excelentes), a serem respondidos em escalas tipo Likert de sete pontos. A consistência interna da escala, no estudo atual foi igual a 0,85 .

\section{Procedimentos}

Os dados foram coletados por meio de questionários impressos $(39,1 \%$ da amostra) ou por meio digital (60,9\% da amostra). Em ambos os casos, os participantes foram inicialmente informados sobre os objetivos do estudo e sobre o sigilo das informações a serem fornecidas, além de que a participação era voluntária. Eles foram abordados em seus locais de trabalho, em ambientes de treinamento ou em salas de aula de cursos de graduação e pós-graduação, assim como por meio das redes sociais. Embora estivessem reunidos em grupos, em alguns desses locais, os participantes responderam ao questionário individualmente em todos os casos.

Para a análise dos dados, foram realizadas análises preliminares para a verificação e tratamento dos casos omissos e extremos, sendo que não houve respostas omissas. Posteriormente, foram efetuadas análises fatoriais confirmatórias para a verificação da estrutura interna da escala, utilizando-se o programa AMOS 18. Os índices de ajuste foram avaliados de acordo com as recomendações de Byrne (2012), para quem um modelo bem ajustado aos dados deve estar de acordo com os seguintes indicadores: $\mathrm{CFI} \geq 0,95$; $\mathrm{TLI} \geq 0,95$; RMSEA $\leq 0,08$. Buscou-se avaliar os parâmetros dos itens por meio da Teoria Clássica dos Testes (TCT) e da Teoria de Resposta ao Item (TRI). Nesse contexto, testou-se o ajuste dos dados ao modelo nominal (Bock, 1972) e de resposta graduada (Samejima, 1969). Os parâmetros da TCC foram calculados com o auxílio do software Excel, e os parâmetros da TRI foram estimados por meio do software Multilog 7.03. Posteriormente, foram realizados os cálculos para a investigação dos coeficientes de correlação e de regressão entre a WAAQ e as demais escalas empregadas no estudo, por meio do programa SPSS.

\section{RESULTADOS}

\section{Modelagem por Equações Estruturais}

Com o intuito de corroborar a unidimensionalidade da escala, foram adotados procedimentos de Análise Fatorial Confirmatória (AFC) destinados a testar o grau de ajuste do modelo teórico do WAAQ aos dados empíricos coletados. Foram testados três diferentes modelos: um modelo principal contendo os sete itens da escala (Modelo 1), um modelo alternativo no qual o item 3 foi excluído em função da multicolinearidade (Modelo 2) e um outro modelo no qual os itens 2 e 3 foram excluídos,também em função da multicolinearidade (Modelo 3). Os dados obtidos evidenciaram que o modelo 1 obteve índices satisfatórios de ajuste, muito embora os demais modelos tenham apresentado índices de ajuste um pouco melhores. Nesse sentido, optou-se pela adoção do Modelo 1, como forma de não desconfigurar o modelo original que norteou a construção do instrumento, o que ainda poderia ocasionar uma diminuição da precisão (Tabela 1).

Cumpre ressaltar também que, conforme apontado na Figura 1, todos os coeficientes de regressão do modelo retido foram estatisticamente significativos e diferentes de zero $(p<0,001)$, além de terem se mostrado de moderados a fortes. Em síntese, a versão brasileira da WAAQ reproduziu integralmente a estrutura unifatorial do instrumento original, tendo ficado composta pelos mesmos sete itens que a compõem. A consistencia interna da escala, calculada por meio do coeficiente Alfa de Cronbach, foi igual a 0,78.

TABELA 1

Índices de ajuste na análise fatorial confirmatória da Escala de Flexibilidade Psicológica no Trabalho (WAAQ)

\begin{tabular}{ccccccc}
\hline & $\chi^{2}(\mathrm{gl})$ & TLI & CFI & RMR & RMSEA (IC) & BIC \\
\hline Modelo 1 & $51,414(14)$ & 0,936 & 0,957 & 0,091 & $0,068(0,048-0,088)$ & 140,569 \\
Modelo 2 & $18,786(9)$ & 0,975 & 0,985 & 0,067 & $0,043(0,014-0,071)$ & 95,204 \\
Modelo 3 & $13,571(5)$ & 0,971 & 0,985 & 0,068 & $0,054(0,020-0,090)$ & 77,253 \\
\hline
\end{tabular}

Notas: Modelo 1= escala completa; Modelo $2=$ excluido o item 3; Modelo $3=$ excluido os itens 2 e 3 . IC $=$ intervalo de confiança. Para todos os modelos, $\mathrm{n}=583$. Nessa tabela, os resultados são apresentados em três casas decimais para facilitar a comparação dos modelos. 


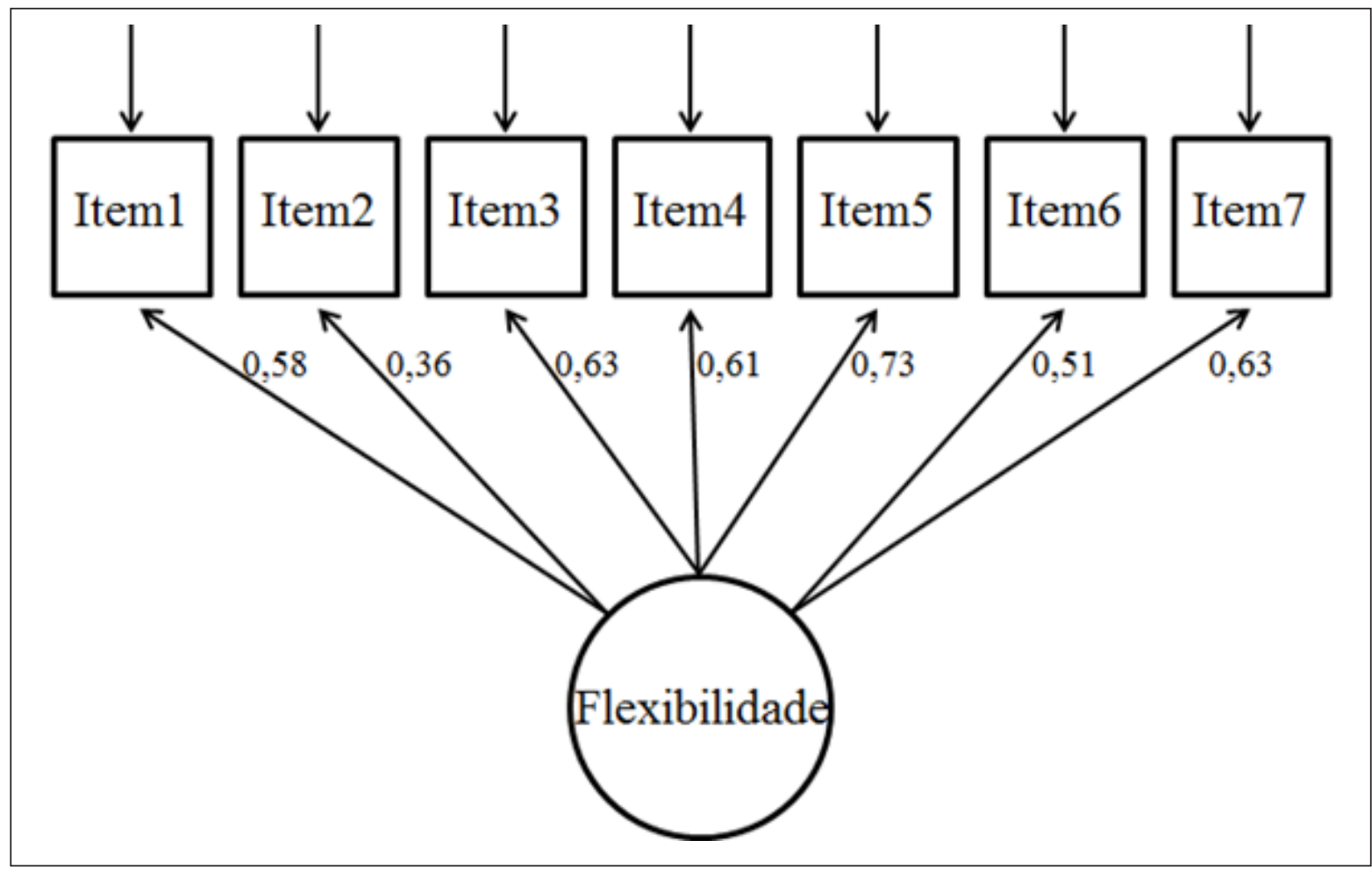

Figura 1. Modelo de equações estruturais da Escala de Flexibilidade Psicológica no Trabalho. As variâncias residuais dos itens foram omitidas na figura.

\section{Análise de Itens}

Além do modelo de estrutura, buscou-se avaliar os parâmetros psicométricos dos itens da Escala de Flexibilidade Psicológica no Trabalho (WAAQ). Para tanto, avaliou-se, primeiramente, sob a perspectiva da Teoria Clássica dos Testes (TCT), a média dos itens e dos escores totais em cada categoria de reposta, bem como a correlação item-total. Tais resultados são apresentados na Tabela 2 e indicam que a maior parte dos itens apresentou média próxima ou levemente acima da mediana teórica (considerando a escala de sete pontos, a mediana teórica é igual a quatro). Assim, esses itens podem ser considerados medianamente endossáveis (ou de dificuldade média). No que se refere à discriminação, todos os itens apresentaram correlações item-total moderadas (Média $r_{\text {it }}=0,65$ ). Buscou-se avaliar, também, a relação entre o endosso em alguma categoria específica e o escore total do participante. Por exemplo, para os participantes que endossaram a categoria 1 do item 1 , a média dos escores totais foi igual a 19,14 . Os resultados indicaram que, de maneira geral, os menores escores totais médios correspondem às categorias 1,2 e 3 , e os maiores escores totais médios estão associados aos participantes que endossaram as categorias 5, 6 e 7 . Ademais, à exceção dos itens 2 e 3 , há uma tendência dos escores totais aumentarem conforme a transição do endosso de uma categoria mais baixa para uma mais alta. Tais resultados evidenciam que os itens e as categorias apresentaram parâmetros de discriminação adequados.

Os parâmetros de dificuldade e discriminação apresentados na Tabela 2 foram calculados no contexto da TCT e, portanto, são dependentes da amostra e dos escores totais. Buscando estimar os parâmetros dos itens de maneira independente dos escores totais, os dados foram analisados por meio dos modelos da Teoria de Resposta ao Item (TRI). Iniciaram-se as análises com o modelo nominal, por apresentar menos restrições aos parâmetros. Esse modelo permite estimar um parâmetro de discriminação para cada categoria de resposta. Além disso, a ordem das categorias não é fixada a priori. Dessa maneira, é possível avaliar se, empiricamente, as categoriais de resposta são endossadas conforme a ordem esperada. Os resultados do modelo nominal são apresentados na parte superior da Tabela 3 e, conforme esperado, os parâmetros de discriminação do modelo nominal variaram do mais baixo (para as primeiras categorias) ao mais alto (para as últimas categorias). Excetuando-se os itens 2 e 3, a discriminação é 
crescente para o contínuo das categorias de resposta. Tais resultados indicam que as categorias mais baixas tenderam, de fato, a serem menos endossadas por participantes com escores latentes (theta) altos, além de que a probabilidade de endosso dessas primeiras categorias diminui conforme o aumento dos escores latentes. Ademais, a probabilidade de endosso das categorias mais altas esteve associada aos thetas mais altos. No que se refere aos interceptos do modelo nominal, os resultados indicaram que a probabilidade de endosso é maior para as categorias 5 e 6 , quando comparadas às demais.

TABELA 2

Médias dos escores dos itens, escores totais médios separados por categoria de reposta e correlações item-total

\begin{tabular}{|c|c|c|c|c|c|c|c|c|c|}
\hline \multirow{2}{*}{ Item } & \multirow{2}{*}{$\begin{array}{c}\text { Média } \\
(D P)\end{array}$} & \multicolumn{7}{|c|}{ Escore total médio e DP separados por participantes que endossaram as seguintes categorias de resposta } & \multirow{2}{*}{$r_{i t}$} \\
\hline & & Categ. 1 & Categ. 2 & Categ. 3 & Categ. 4 & Categ. 5 & Categ. 6 & Categ. 7 & \\
\hline 1 & $5,02(1,39)$ & $19,14(5,52)$ & $22,77(6,11)$ & $26,29(5,56)$ & $29,88(4,86)$ & $32,18(5,82)$ & $35,80(5,56)$ & $39,64(6,37)$ & 0,63 \\
\hline 2 & $5,65(1,29)$ & $23,67(10,25)$ & $23,22(7,92)$ & $23,04(5,29)$ & $28,60(5,68)$ & $30,96(5,60)$ & $33,45(6,19)$ & $36,60(7,04)$ & 0,48 \\
\hline 3 & $4,56(1,53)$ & $24,29(5,74)$ & $23,24(5,41)$ & $27,90(5,23)$ & $31,03(5,01)$ & $33,54(4,79)$ & $37,55(5,11)$ & $42,02(6,47)$ & 0,69 \\
\hline 4 & $4,04(1,66)$ & $23,67(5,43)$ & $26,28(6,48)$ & $30,18(4,79)$ & $32,04(5,08)$ & $35,80(4,87)$ & $38,79(4,97)$ & $40,77(6,27)$ & 0,68 \\
\hline 5 & $4,80(1,61)$ & $22,24(6,39)$ & $23,71(4,66)$ & $25,71(4,49)$ & $30,19(4,69)$ & $32,53(4,59)$ & $36,97(4,62)$ & $40,88(5,19)$ & 0,75 \\
\hline 6 & $4,44(1,75)$ & $24,79(6,82)$ & $26,34(5,81)$ & $27,56(5,67)$ & $31,93(5,09)$ & $34,42(4,98)$ & $36,16(5,72)$ & $40,06(6,26)$ & 0,63 \\
\hline 7 & $4,17(1,78)$ & $24,79(6,05)$ & $26,72(5,77)$ & $28,47(4,45)$ & $31,15(4,56)$ & $35,35(5,18)$ & $38,06(4,80)$ & $41,09(5,53)$ & 0,70 \\
\hline
\end{tabular}

$D P=$ desvio-padrão; Categ $=$ categoria de resposta; $r_{\text {it }}=$ correlação item-total .

TABELA 3

Parâmetros dos itens estimados por modelos de TRI

\begin{tabular}{|c|c|c|c|c|c|c|c|c|c|c|c|c|c|c|}
\hline \multicolumn{15}{|c|}{ Modelo Nominal (constrained parameters) } \\
\hline Item & $\alpha_{j 1}$ & $\alpha_{j 2}$ & $\alpha_{j 3}$ & $\alpha_{j 4}$ & $\alpha_{j 5}$ & $\alpha_{j 6}$ & $\alpha_{j 7}$ & $\gamma_{j 1}$ & $\gamma_{j 2}$ & $\gamma_{j 3}$ & $\gamma_{j 4}$ & $\gamma_{j 5}$ & $\gamma_{j 6}$ & $\gamma_{j 7}$ \\
\hline 1 & $-1,81$ & $-1,37$ & $-0,6$ & 0,01 & 0,48 & 1,21 & 2,09 & $-3,27$ & $-1,35$ & 0,27 & 0,89 & 1,62 & 1,74 & 0,11 \\
\hline 2 & $-0,25$ & $-0,63$ & $-0,97$ & 0,03 & 0,26 & 0,53 & 1,03 & $-1,57$ & $-1,84$ & $-1,21$ & 0,13 & 1,25 & 1,85 & 1,39 \\
\hline 3 & $-1,08$ & $-1,96$ & $-0,92$ & $-0,32$ & 0,32 & 1,35 & 2,62 & $-1,22$ & $-0,81$ & 0,41 & 0,57 & 1,63 & 0,90 & $-1,48$ \\
\hline 4 & $-1,63$ & $-1,28$ & $-0,45$ & $-0,30$ & 0,64 & 1,24 & 1,78 & $-1,14$ & 0,08 & 0,64 & 0,7 & 0,86 & $-0,06$ & $-1,09$ \\
\hline 5 & $-1,99$ & $-1,98$ & $-1,58$ & $-0,29$ & 0,57 & 2,05 & 3,22 & $-2,01$ & $-0,95$ & $-0,08$ & 0,56 & 1,51 & 1,47 & $-0,50$ \\
\hline 6 & $-0,91$ & $-0,83$ & $-0,87$ & 0,08 & 0,43 & 0,70 & 1,40 & $-0,76$ & $-0,36$ & $-0,17$ & 0,36 & 0,79 & 0,61 & $-0,46$ \\
\hline 7 & $-1,37$ & $-1,2$ & $-1,06$ & $-0,55$ & 0,69 & 1,35 & 2,14 & $-0,72$ & 0,19 & 0,09 & 0,4 & 0,82 & 0,28 & $-1,06$ \\
\hline
\end{tabular}

Ajuste: $-2 \log (\mathrm{gl})=6006,3(84) ; \mathrm{BIC}=6541,23$.

\begin{tabular}{|c|c|c|c|c|c|c|c|}
\hline \multicolumn{8}{|c|}{ Modelo de Resposta Graduada } \\
\hline Item & $\alpha_{j}(E P)$ & $\delta_{j l}(E P)$ & $\delta_{j 2}(E P)$ & $\delta_{j 3}(E P)$ & $\delta_{j 4}(E P)$ & $\delta_{j 5}(E P)$ & $\delta_{j 6}(E P)$ \\
\hline 1 & $1,40(0,12)$ & $-3,75(0,43)$ & $-2,50(0,23)$ & $-1,49(0,14)$ & $-0,77(0,10)$ & $0,26(0,09)$ & $1,91(0,19)$ \\
\hline 2 & $0,79(0,10)$ & $-5,65(0,94)$ & $-4,72(0,72)$ & $-3,53(0,49)$ & $-2,45(0,35)$ & $-0,81(0,17)$ & $1,46(0,26)$ \\
\hline 3 & $1,68(0,12)$ & $-2,76(0,25)$ & $-1,62(0,14)$ & $-0,89(0,10)$ & $-0,42(0,08)$ & $0,77(0,10)$ & $2,14(0,19)$ \\
\hline 4 & $1,47(0,12)$ & $-2,40(0,22)$ & $-1,19(0,13)$ & $-0,43(0,09)$ & $0,25(0,09)$ & $1,28(0,13)$ & $2,26(0,21)$ \\
\hline 5 & $2,18(0,14)$ & $-2,42(0,19)$ & $-1,56(0,11)$ & $-0,89(0,08)$ & $-0,49(0,07)$ & $0,26(0,07)$ & $1,54(0,11)$ \\
\hline 6 & $1,12(0,10)$ & $-2,70(0,29)$ & $-1,71(0,19)$ & $-0,95(0,14)$ & $-0,22(0,11)$ & $0,84(0,13)$ & $2,23(0,24)$ \\
\hline 7 & $1,51(0,12)$ & $-2,21(0,20)$ & $-1,12(0,12)$ & $-0,55(0,09)$ & $0,02(0,09)$ & $0,93(0,11)$ & $2,03(0,18)$ \\
\hline
\end{tabular}

Ajuste: $-2 \log (\mathrm{gl})=6012,8(49) ; \Delta-2 \log (\Delta \mathrm{gl})=6,5(35) ; \mathrm{BIC}=6324,84$

Notas: $\alpha_{\mathrm{j}}=$ parâmetro de discriminação; $\gamma_{\mathrm{j}}=$ intercepto; $\delta_{\mathrm{j}}=$ parâmetro de transição entre categorias $($ threshold $)$; $-2 \log =-2$ log likelihood; gl= graus de liberdade; $\mathrm{BIC}=$ Bayesian Information Criterion; $\Delta-2 \log (\Delta \mathrm{gl})=$ diferença do ajuste e dos graus de liberdade entre os modelos nominal e de resposta graduada. Para todos os modelos, $\mathrm{n}=583$. 
Em resumo, os resultados do modelo nominal sugeriram que, de maneira geral, as categorias de resposta parecem funcionar de maneira ordinal, ou seja, o aumento do escore latente respalda o aumento da probabilidade de transição de uma categoria mais baixa para uma categoria mais alta. Contudo, por impor poucas restrições aos dados, no modelo nominal, uma quantidade grande de parâmetros (84, no total) é estimada, o que pode gerar instabilidade no modelo. De maneira alternativa é possível estimar apenas um parâmetro de discriminação para cada item, fixando a ordem das categorias de resposta. Tal alternativa é característica do modelo de resposta graduada (Graded Response Model-GRM). Os resultados desse modelo são apresentados na parte inferior da Tabela 3. Considerando que o GRM é mais restrito do que o modelo nominal, esperava-se uma diminuição do ajuste do modelo aos dados. No entanto, verifica-seque a diferença dos indicadores de ajuste $(\Delta-2 \log )$ entre esses modelos foi de apenas 6,5 unidades logarítmicas de máxima verossimilhança. Tal diferença, ponderando o $\Delta \mathrm{gl}$ igual a 35 , não é estatisticamente significativa ( $p<0,05$, para uma distribuição de qui-quadrado). Ademais, o indicador de ajuste BIC apresentou valor inferior para o GRM se comparado ao nominal, indicando que, ao penalizar o modelo com mais parâmetros estimados, o GRM ajustou-se melhor aos dados. Em outras palavras, a estimação de um modelo mais restrito é tão plausível (ou melhor ajustado) quanto a estimação do modelo nominal. Tal resultado também indica que as categorias de resposta podem ser consideradas de maneira crescente.

Cabe ainda ressaltar que a discriminação da maior parte dos itens foi adequada (Média de $\alpha=1,45$ ). Nesse sentido, somente o item 2 apresentou discriminação abaixo de 1. Os parâmetros de transição, por sua vez, apontam para itens relativamente fáceis de serem endossados. Assim, por exemplo, um theta próximo de 2 é suficiente para que a categoria 7 seja a opção mais provável de resposta.

\section{Relação com Variáveis Externas}

Buscou-se também avaliar a relação da flexibilidade psicológica com variáveis teoricamente correlacionadas. A Tabela 4 apresenta as médias, os desvios padrões e as correlações entre as diferentes escalas adotadas no estudo. Como previsto, a flexibilidade psicológica no trabalho apresentou-se positiva e significativamente correlacionada com o engajamento no trabalho $(r=0,30 ; p<0,01)$; a saúde mental $(r=0,28 ; p<0,01)$; a abertura $(r=0,15 ; p<0,01)$ e a satisfação com a vida $(r=0,25 ; p<0,01)$. Também conforme esperado, a flexibilidade psicológica no trabalho apresentou-se negativa e significativamente correlacionada com o neuroticismo $(r=-0,29$; $p<0,01)$.

O poder preditivo da flexibilidade psicológica sobre o engajamento no trabalho, controlando-se o efeito dos fatores de personalidade abertura à experiência e neuroticismo, foi também analisado. Os modelos de regressão indicaram que as dimensões de personalidade explicaram $11,1 \%$ da variância do engajamento do trabalho, sendo que a flexibilidade psicológica explicou mais $4,6 \%$ da variância de tal variável critério. Todos os coeficientes de regressão foram estatisticamente significativos $(p<0,05)$, porém baixos. Os valores de beta para abertura à experiência, neuroticismo e flexibilidade foram iguais a $0,17,-0,18$ e 0,23, respectivamente. Tais resultados indicam que a flexibilidade psicológica é capaz de prever, de maneira fraca, os resultados de engajamento no trabalho, mesmo quando controlado o efeito da personalidade.

TABELA 4

Relações entre a Flexibilidade Psicológica e demais variáveis do estudo

\begin{tabular}{|c|c|c|c|c|c|c|c|}
\hline & $M$ & $D P$ & 1 & 2 & 3 & 4 & 5 \\
\hline 1. Flexibilidade psicológica no trabalho & 4,67 & 1,04 & & & & & \\
\hline 2. Engajamento no trabalho & 4,08 & 1,26 & 0,30 & & & & \\
\hline 3. Questionário de Saúde Geral & 2,93 & 0,48 & 0,28 & 0,32 & & & \\
\hline 4. Abertura à Experiência & 3,80 & 0,59 & 0,15 & 0,23 & 0,11 & & \\
\hline 5. Neuroticismo & 2,92 & 0,88 & $-0,29$ & $-0,28$ & $-0,43$ & $-0,17$ & \\
\hline 6. Satisfação com a vida & 4,61 & 1,30 & 0,25 & 0,35 & 0,51 & 0,17 & $-0,25$ \\
\hline
\end{tabular}




\section{DISCUSSÃO}

O objetivo do presente trabalho foi adaptar e reunir evidências iniciais de validade da Escala de Avaliação da Flexibilidade Psicológica no Ambiente do Trabalho (WAAQ; Bond et al., 2013), em amostras brasileiras. Após a realização das análises fatoriais confirmatórias, optou-se pela adoção de um modelo unifatorial que reproduzia fielmente o modelo original da escala, em função de ele ter apresentado índices de ajuste satisfatórios. O índice de consistência interna da escala, calculado por meio do Alfa de Cronbach, foi também satisfatório $(\alpha=0,78)$. Tais resultados mostram-se consistentes com os achados de Ruiz e OdriozolaGonzález (2014), que também confirmaram a estrutura unifatorial da escala em amostras espanholas. Cabe ressaltar que o item 2 apresentou carga fatorial abaixo do esperado (coeficiente pattern padronizado igual a 0,36 ), sendo que no estudo original da escala (Bond et al., 2013) uma carga fatorial baixa também foi observada nesse item. Uma possível explicação para esse resultado pode se dever ao conteúdo dos itens, na medida em que o item 2 encontra-se mais associado ao comportamento de assumir e admitir erros, enquanto os demais itens estão mais relacionados a sentimentos e preocupações.

Os parâmetros dos itens da WAAQ foram estimados por meio da Teoria Clássica dos Testes (TCT) e da Teoria de Resposta ao Item (TRI). De maneira geral, em todos os modelos, a discriminação foi estimada de maneira adequada para a maior parte dos itens. Esse aspecto é importante do ponto de vista prático, pois garante que o instrumento é capaz de diferenciar participantes com escores latentes próximos. No que se refere aos parâmetros de localização e transição de uma categoria para outra (thresholds), tanto o modelo menos restrito (nominal) quanto o mais restrito (resposta graduada) indicaram que as categorias de resposta funcionaram de maneira ordinal e crescente. Esse aspecto é relevante para a utilização da escala em pesquisas, uma vez que garante que os participantes com menores escores latentes tenham, de fato, maior probabilidade de endossarem as categorias mais baixas, e que participantes com escores latentes altos tendam a endossar as categorias mais altas.

Destaca-se, ainda, que o item dois apresentou discriminação inferior ao esperado. Por um lado, esse resultado não pode ser comparado com os estudos originais da escala (Bond et al., 2011, 2013), uma vez que os autores não modelaram os dados por meio da TRI. Por outro lado, sabendo que a discriminação dos itens apresenta forte associação às cargas fatoriais, a baixa discriminação do item dois é coerente com os resultados do estudo original, ou seja, a variável latente flexibilidade explica menos variância do item dois se comparada à variância explicada dos demais itens. Além da pequena diferença de conteúdo entre esse item e os demais, já discutida no presente estudo, devese atentar para a diferença de dificuldade de endosso. Nesse contexto, para a maior parte dos itens, thetas entre -2 e -3 respaldam probabilidades equivalentes de endosso entre as categorias um e dois, bem como entre as categorias dois e três. Em outras palavras, a transição de uma categoria mais baixa para uma menos baixa ocorre em pontos baixos da escala theta. Especificamente para o item dois, essa transição ocorre em níveis ainda mais baixos da escala. Ou seja, é necessário um escore de flexibilidade muito abaixo da média (abaixo de -4 ou -5) para que a probabilidade de endosso das categorias um e dois seja alta. Como esse fenômeno ocorreu apenas para o item dois, é possível que a baixa discriminação esteja associada, também, a um fator espúrio de dificuldade. Um ajuste no conteúdo do item, no intuito de deixá-lo levemente mais difícil, poderia resolver esse problema. Contudo, obviamente, tal alteração deve ser realizada com a anuência dos autores da escala original.

No que diz respeito à correlação da WAAQ com outros construtos a ela relacionados, verificou-se que a escala em questão apresentou correlação positiva com o engajamento no trabalho. Foi demonstrado assim que as pessoas que respondem mais eficazmente às contingências do contexto laboral que costumam impedir a realização de metas, sem medo de tomar iniciativa, costumam se mostrar também mais integradas e motivadas com seu trabalho e com a organização (Bond et al., 2013). Tais dados vão ao encontro do estudo de Bond et al. (2013), que tambem obteve correlação positiva e significativa entre a flexibilidade psicologica no trabalho e o engajamento.

Uma correlação positiva foi também observada entre a flexibilidade psicológica e a saúde mental. Desse modo, o fato de os indivíduos serem capazes de perceber o grau em que detêm o controle sobre assituações, comportando-se de acordo com seus próprios valores e tentando resolvê-las sem se esquivar (Hayes et al., 2006), pode contribuir para a promoção de sua saúde mental, conforme observado por Bond e Bunce (2000) no contexto do trabalho.

O resultado sobre a correlação positiva da flexibilidade psicológica com a abertura converge com o estudo de Bond et al. (2013), que tambem encontraram uma correlação positiva entre a flexibilidade psicologica no trabalho e a abertura. Tais dados indicam que quando as pessoas se comportam de acordo com seus valores, 
sem se deixar levar pelos sentimentos positivos e negativos momentâneos despertados por tal situação, elas se tornam mais abertas, receptivas e curiosas e, assim, reconhecem e buscam novos conhecimentos e experiências.

No que diz respeito à correlação positiva entre a flexibilidade psicológica e a satisfação com a vida, não foram encontrados estudos anteriores sobre tal relação. De todo modo, as evidências ora obtidas sinalizam que os indivíduos que perseguem seus valores e metas, sem se deixar levar por experiências internas ou contingências situacionais que possam se interpor nessa trajetória, tendem a ter uma melhor qualidade de vida e bem-estar, expressa em maior satisfação com a vida que levam.

Atendo-se à correlação negativa do neuroticismo com a flexibilidade psicologica, é possível afirmar que as pessoas com niveis mais baixos de autoestima, tensão e mal humor tendem a resistir à modificação de seus comportamentos, mesmo quando existe uma evidência clara de que estão realizando a tarefa ineficazmente, em função de sua incapacidade de separar os pensamentos e sentimentos negativos e se focalizar na situação presente, de modo a atingir suas metas de trabalho. Tais dados mostram-se consistentes com os de Bond et al. (2013), que tambem constataram uma correlação negativa e significativa entre o neuroticismo e a flexibilidade psicologica no trabalho. Cumpre ressaltar, porém, que as correlações encontradas tanto no presente estudo quanto na pesquisa de Bond et al. (2013) foram, no máximo, moderadas.

Foi também constatado na análise de regressão que a flexibilidade psicológica mostrou-se capaz de prever os resultados de engajamento no trabalho, mesmo quando controlado o efeito da personalidade, ainda que de maneira fraca. Esses achados vão ao encontro dos resultados anteriores de Bond et al. (2013) e indicam que a flexibilidade psicológica explica variância do engajamento do trabalho não explicada por fatores de personalidade, o que recomenda a utilização do WAAQ no contexto laboral.

Em síntese, os resultados das análises fatoriais confirmatórias, dos parâmetros dos itens e do Alfa de Cronbach, aliados aos obtidos nas correlações da WAAQ com diversas variáveis, evidenciam que a escala apresentou boas características psicométricas, no que diz respeito a sua consistência interna e seus indicadores de validade de construto. Tais achados recomendam, portanto, o uso futuro da WAAQ em situações de pesquisa da capacidade de as pessoas perceberem e responderem eficazmente às contingências relacionadas ao desempenho laboral, adotando ações direcionadas à consecução de suas metas laborais, ainda que na presença de experiências internas de difícil aceitação. Considerando-se o fato de que o processo de flexibilidade psicológica pode atuar de forma específica no contexto do trabalho, ele poderia ser melhor diagnosticado por uma medida de flexibilidade psicológica adaptada a tal contexto particular, o que poderá trazer beneficios às organizações que desejarem avaliar a flexibilidade de seus empregados para fins especificos (Bond et al., 2011).

Cumpre ressaltar, porém, a necessidade de tal construto ser mais explorado do ponto de vista empírico. Dessa forma, a relação da flexibilidade psicológica no trabalho com outras variáveis do contexto organizacional, bem como com atitudes e comportamentos organizacionais, poderiam ser testadas.Nesse sentido, pesquisas futuras poderiam investigar o papel que as demandas (como a sobrecarga) ou recursos (como autonomia) do contexto laboral exercem sobre a flexibilidade psicológica no trabalho. Outrossim, os efeitos de tal construto no comprometimento e na cidadania organizacional também mereceriam ser objeto de estudos posteriores.

No que tange às limitações do presente estudo, o fato de todas as medidas utilizadas terem sido de autorrelato podem ter introduzido efeitos de desejabilidade social nos dados. Outro ponto que deve ser enfatizado refere-se à generalização dos resultados, que foram coletados apenas nas cidades do Rio de Janeiro e Niterói e, podem, assim, estar relacionados ao contexto expecífico dessas duas cidades. Acrescente-se a isso o fato de que houve um equívoco na tradução da âncora da primeira categoria (never true) da escala de flexibilidade psicológica. No entanto, as análises de itens com modelos de TCT e TRI indicaram, entre outros aspectos, que as categorias funcionaram conforme o esperado, a despeito do equívoco de tradução. Tornam-se porém necessários mais estudos, com uma versão da escala com tal equívoco corrigido, a qual é disponibilizada ao final do artigo, e utilizando-se de amostras com diferentes tipos de trabalhadores, pertencentes a diferentes setores e organizações, que permitam maior generalização dos dados ora obtidos. 


\section{REFERENNCIAS}

Andrade, J. M. (2008). Evidências de validade do Inventário dos Cinco Grandes Fatores de Personalidade para o Brasil (Tese de doutorado). Universidade de Brasília, Brasília, DF, Brasil.

Bakker, A., Demerouti, E., \& Sanz-Vergel, A. I. (2014). Burnout and work engagement: The JD-R approach. Annual Review of Organizational Psychology and Organizational Behavior, 1, 389-411. http://dx.doi.org/10.1146/annurevorgpsych-031413-091235

Bock, R. D. (1972). Estimating item parameters and latent ability when responses are scored in two or more nominal categories. Psychometrika, 37, 29-51. http://dx.doi.org/10.1007/BF02291411

Bond, F. W., \& Bunce, D. (2000). Mediators of change in emotion-focused and problem-focused worksite stress management interventions. Journal of Occupational Health Psychology, 5, 156-163. http://dx.doi.org/10.1037/10768998.5.1.156

Bond, F. W., Flaxman, P. E., van Veldhoven, M. J. P. M., \& Biron, M. (2010). The impact of psychological flexibility and acceptance and commitment therapy (ACT) on health and productivity at work. In J. Houdmont \& S. Leka (Eds.). Contemporary occupational health psychology: Global perspectives on research, education, and practice (pp. 296-313). Chichester, UK: Wiley-Blackwell. http://dx.doi.org/10.1002/9780470661550.ch15

Bond, F. W., Hayes, S. C., Baer, R. A., Carpenter, K. M., Guenole, N., Orcutt, H. K., Waltz, T., \& Zette, R. D. (2011). Preliminary psychometric properties of the Acceptance and Action Questionnaire-II: A revised measure of psychological inflexibility and experiential avoidance. Behavior Therapy, 42, 676-688. http://dx.doi.org/10.1016/j. beth.2011.03.007

Bond, F. W., Lloyd, J., \& Guenole, N. (2013). The work-related acceptance and action questionnaire: Initial psychometric findings and their implications for measuring psychological flexibility in specific contexts. Journal of Occupational and Organizational Psychology, 86, 331-347. http://dx.doi.org/10.1111/joop.12001

Diener, E., Emmons, R. A., Larsen, R. J., \& Griffin, S. (1985). The Satisfaction With Life Scale. Journal of Personality Assessment, 49, 71-75. http://dx.doi.org/10.1207/s15327752jpa4901_13

Byrne, B. M. (2012). Structural equation modeling with Mplus: Basic concept, applications, and programming. New York, NY: Routledge.

Ferreira, M. C., Valentini, F., Mourão, L., Porto, J., Oliveira, D., \& Monteiro, A. C. (2014). Evidencias de validade da Escala de Engajamento no Trabalho de Utrecht em amostras brasileiras. Manuscrito submetido à publicação.

Flaxman, P. E. \& Bond, F. W. (2010). A randomised worksite comparison of acceptance and commitment therapy and stress inoculation training. Behaviour Research and Therapy, 48, 816-820. http://dx.doi.org/10.1016/j.brat.2010.05.004

Friedman, H. S. \& Schustack, M. W. (2004). Teorias da personalidade. Da teoria clássica à pesquisa moderna (2ª ed.). São Paulo: Prentice Hall.

Goldberg, D. (1972). The detection of psychiatric illness by questionnaire. London, UK: Oxford University Press. http:// dx.doi.org/10.1192/bjp.122.4.483

Gouveia, V. V., Barbosa, G. A., Andrade, E. O., \& Carneiro, M. B. (2005). Medindo a satisfação com a vida dos médicos no Brasil. Jornal Brasileiro de Psiquiatria, 54, 298-305.

Hayes, S. C., Luoma, J. B., Bond, F. W., Masuda, A., \& Lillis, J. (2006). Acceptance and commitment therapy: Model, processes and outcomes. Behaviour Research and Therapy, 44, 1-25. http://dx.doi.org/10.1016/j.brat.2005.06.006

Hayes, S. C., Levin, M. E., Plumb-Vilardaga, J., Villatte, J. L., \& Pistorello, J. (2013). Acceptance and commitment therapy and contextual behavioral science: Examining the progress of a distinctive model of behavioral and cognitive therapy. Behavior Therapy, 44, 180-198. http://dx.doi.org/10.1016/j.beth.2009.08.002

Hayes, S. C., Strosahl, K. D., \& Wilson, K. G. (1999). Acceptance and commitment therapy: An experiential approach to behavior change. New York: Guilford Press.

Hayes, S. C., Strosahl, K. D., Wilson, K. G., Bissett, R., Pistorello, J., Toarmino, D., \& McCurry, S. M. (2004). Measuring experiential avoidance: A preliminary test of a working model. The Psychological Record, 54, 553-578.

John, O. P. \& Srivastava, S. (1999). The Big-Five trait taxonomy: History, measurement, and theoretical perspectives. In L. A. Pervin \& O. P. John (Eds.). Handbook of personality: Theory and research (Vol. 2; pp. 102-138). New York, NY: Guilford Pess.

John, O. P., Donahue, E. M., \& Kentle, R. L. (1991). The Big Five Inventory: Versions 4a and 54. Berkeley, CA: University of California, Berkeley, Institute of Personality and Social Research.

Kashdan, T. B. \& Rottenberg, J. (2010). Psychological flexibility as a fundamental aspect of health. Clinical Psychology Review, 30, 865-878. http://dx.doi.org/10.1016/j.cpr.2010.03.001

Pasquali, L., Gouveia, V. V., Andriola, W. B., Miranda, F. J., \& Ramos, A. L. M. (1994). Questionário de Saúde Geral de Goldberg (QSG): Adaptação brasileira. Psicologia: Teoria e Pesquisa, 10, 421-437.

Ruiz, F. J. \& Odriozola-González, P. (2014). Spanish version of the Work-related Acceptance and Action Questionnaire (WAAQ). Psicothema, 26, 63-68. http://dx.doi.org/10.7334/psicothema2013.110

Samejima, F. A. (1969). Estimation of latent ability using a response pattern of graded scores. Psychometric Monograph, 17. Richmond, VA: Psychometric Society. 
Schaufeli, W. B. \& Bakker, A. B. (2003). UWES - Utrecht Work Engagement Scale: Test Manual. Utrecht University, Department of Psychology. Retirado em 11 out. 2012, de <http://www.wilmarschaufeli.nl/publications/Schaufeli/ Tests/ UWES P S 9.pdf>.

Seligman, M. E. P. \& Csikszentmihalyi, M. (2000). Positive psychology: An introduction. American Psychologist, 55, 5-14. http://dx.doi.org/10.1037/0003-066X.55.1.5

\section{ESCALA DE FLEXIBILIDADE PSICOLÓGICA NO TRABALHO}

As frases a seguir referem-se a seu modo de se relacionar com seu trabalho. Para expressar suas opiniões sobre as mesmas, utilize a escala abaixo, indicando a frequência que melhor lhe descreveria marcando um $X$ na coluna do número que melhor corresponde à sua opinião:

\begin{tabular}{|c|c|c|c|c|c|c|}
\hline 1 & 2 & 3 & 4 & 5 & 6 & 7 \\
\hline $\begin{array}{c}\text { Nunca } \\
\text { verdadeiro }\end{array}$ & $\begin{array}{c}\text { Raramente } \\
\text { verdadeiro }\end{array}$ & $\begin{array}{c}\text { Às vezes, } \\
\text { mas raramente } \\
\text { verdadeiro }\end{array}$ & Neutro & $\begin{array}{c}\text { As vezes } \\
\text { verdadeiro }\end{array}$ & $\begin{array}{c}\text { Normalmente } \\
\text { verdadeiro }\end{array}$ & $\begin{array}{c}\text { Sempre } \\
\text { verdadeiro }\end{array}$ \\
\hline
\end{tabular}

\begin{tabular}{|c|c|c|c|c|c|c|c|}
\hline & 1 & 2 & 3 & 4 & 5 & 6 & 7 \\
\hline \multicolumn{8}{|c|}{ Sou capaz de trabalhar efetivamente, apesar de quaisquer preocupações pessoais que eu tenha. } \\
\hline \multicolumn{8}{|l|}{ Posso admitir meus erros no trabalho e ainda assim ser bem sucedido. } \\
\hline \multicolumn{8}{|l|}{ Posso ainda trabalhar de forma eficaz, mesmo estando nervoso com algo. } \\
\hline \multicolumn{8}{|l|}{ Preocupações não entram em meu caminho para o sucesso. } \\
\hline \multicolumn{8}{|c|}{ Posso executar meu trabalho conforme o necessário, independentemente de como eu me sinta. } \\
\hline \multicolumn{8}{|l|}{ Posso trabalhar de forma eficaz, mesmo quando duvido de mim mesmo. } \\
\hline Meus pensamentos e sentimentos não entram no caminho do meu trabalh & & & & & & & \\
\hline
\end{tabular}

Autores:

Vladimir PINTO NovaES - Mestre em Psicologia, Universidade Salgado de Oliveira

MARIA CRISTINA FERREIRA - Doutor, Universidade Salgado de Oliveira.

FELIPE VALENTINI - Doutor, Universidade Salgado de Oliveira.

Endereço para correspondência:

Vladimir Novaes Pinto

Rua Joaquim Távora, 130 apto 607 - Icaraí

CEP 24230-540 Niterói, RJ, Brasil

Recebido em: 24.09.14

Aceito em: 02.03 .15 